\title{
Optical Channel Dropping using Dielectric Microspheres
}

\author{
Temel Bilici ${ }^{\dagger}$, Senol Isçi ${ }^{*}$, Adnan Kurt, and Ali Serpengüzel ${ }^{\ddagger}$ \\ Koç University, Microphotonics Research Laboratory, Physics Department, \\ Rumeli Feneri Yolu, Sariyer, Istanbul 34450 Turkey
}

\begin{abstract}
Dielectric microspheres, with their morphology dependent resonances, are used to resonantly couple light from half optical fiber couplers. The dropped channels are observed in the elastic scattering and the transmission spectra. The excitation of the microsphere with the nearly Gaussian beam in the half optical fiber coupler provides spatially and spectrally selective, and enhanced light coupling. The filter drops approximately $10 \%(0.5 \mathrm{~dB})$ of the power at the resonance wavelength. A tunable single mode distributed feedback diode laser is used as the infrared excitation source. The generalized Lorenz-Mie theory, describing the illumination of the microsphere with a Gaussian beam, is used to interpret the experimental results.
\end{abstract}

Keywords: Channel dropping filter, integrated optoelectronics, microsphere resonator, morphology dependent resonances, whispering gallery modes.

\section{INTRODUCTION}

Optical microsphere resonators have found various applications in quantum optics, laser science, spectroscopy, and optoelectronics [1]. microspheres possess high quality (Q) factor optical morphology dependent resonances (MDR's) in relatively small volumes [2]. These tiny optical cavities, whose diameters may vary from a few to several hundred micrometers [3]. Low threshold lasing in Nd-doped silica [4], polymer [5] and Raman active media [6] have been demonstrated using microspheres. Strain tunable microsphere oscillators [7], add-drop filters [8], and thermooptical switching in microspheres [9] have also been realized for filtering, multiplexing, and switching applications. MDR's of dielectric microspheres provide the necessary optical feedback for applications in channel dropping for optical communications, such as optical couplers, optical amplifiers, and optical filter, which support narrow channel spacing, high channel counts, and adding / dropping of a large number of wavelengths. In this paper, MDR's are characterized experimentally by evanescently coupling light along an optical fiber half coupler (OFHC) into glass microspheres for possible channel dropping applications.

\section{MORPHOLOGY DEPENDENT RESONANCES}

As the laser propagates around the inside surface of the microsphere, it undergoes an almost total internal reflection (TIR) [10]. The rays of the MDR have the property such that all subsequent bounces have the same angle of incidence. After circumnavigating the microsphere, the laser returns to its starting point in phase to interfere constructively with itself. This constructive interference can occur only at certain discrete MDR wavelengths [11]. Hence, the laser is confined to a band within the great circle of the microsphere. Each MDR is characterized by a mode number (n) and a mode order (I). Physically, (n) indicates the number of nodes in the internal intensity distribution as the polar angle is varied from $0^{\circ}$ to $180^{\circ}$. The mode order (I) indicates the number of nodes in the internal intensity distribution in the radial direction [12]. For each set of MDR, there is a transverse electric (TE) and transverse magnetic (TM) MDR [13]. For a

$\dagger$ Temel Bilici is also with Bogazici University, Department of Electrical and Electronics Engineering, Bebek, Istanbul 34342 Turkey. *Senol Isçi is also with Bogazici University, Biomedical Engineering Institute, Bebek, Istanbul 34342 Turkey. $\ddagger$ Correspondence should be addressed to Ali Serpengüzel, aserpenguzel@ku.edu.tr; phone: + 90 (212) 3381312, fax: + 90 (212) 338-1547, http://home.ku.edu.tr/ aserpenguzel, Koç University, Microphotonics Research Laboratory, Physics Department, Rumeli Feneri Yolu, Sariyer, Istanbul 34450 Turkey. 
given microsphere, the MDR occurs at a specific value of the size parameter, $\mathbf{x}_{\mathbf{n}, \mathbf{l}}$. These MDR's have been verified experimentally at optical wavelengths with micrometer-sized spheres.

Many applications of the microspheres require coupling of light into MDR's from external light sources. The greatest impediment to the use of microsphere resonators in practical devices has been the difficulty of efficiently light coupling into and out of the microspheres. One way to excite MDR's is to use evanescent coupling method. To couple light into or out of the microsphere, it is necessary to overlap the evanescent field of the MDR with the evanescent field of the TIR. Such coupling has been implemented by using optical fiber half coupler (OFHC). If the microsphere is placed on the exposed surface near the evanescent field of the fiber-optic core, then there is an efficient energy exchange between the waveguide mode of the fiber and the MDR of the microsphere [14].

\section{EXPERIMENTAL SETUP}

The schematic of the experimental setup is shown in Fig. 1. The laser is coupled into the microsphere by using the OFHC. The OFHC is fabricated from a $810 \mathrm{~nm}$ single mode fiber, which is laid on a glass substrate with a low curvature [15]. The cladding of the fiber below the microsphere is shaved in order to approach the core of the fiber. This excitation geometry effectively becomes the optical equivalent of a Gaussian beam with an infinite skirt length passing near the microsphere. The transmission loss from the OFHC is $0.8 \%$, corresponds to $21 \mathrm{~dB}$. BK7 type glass microspheres are used in the experiment, because of exhibiting low loss at optical communication wavelengths.

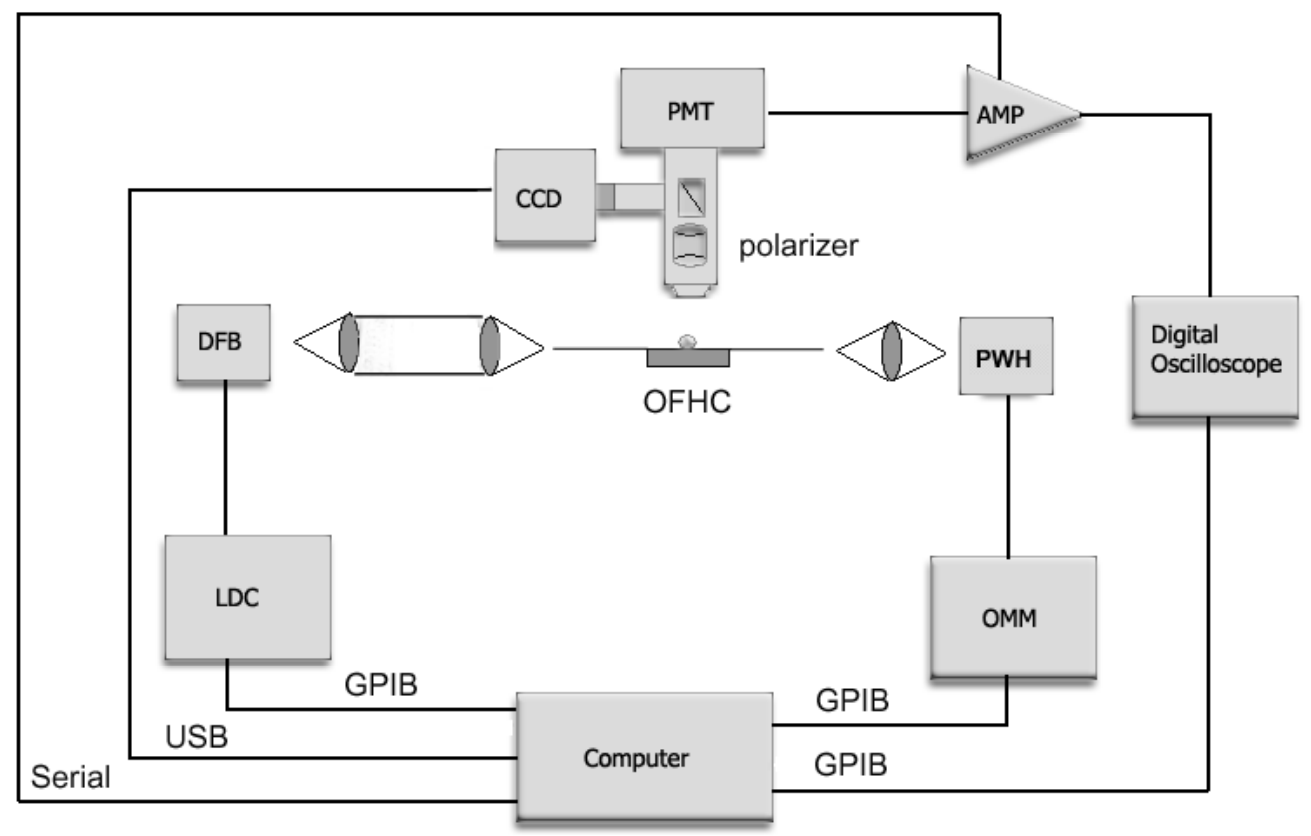

Figure 1. The schematic of the experimental setup.

The glass microsphere has a radius of $0.5 \mathrm{~mm}$ and refractive index of 1.50. Excitation of MDR's of the microsphere is achieved by using a tunable distributed feedback (DFB) semiconductor laser, whose center wavelength is $810 \mathrm{~nm}$. Wavelength tuning is achieved by tuning the temperature of the DFB laser with a laser diode controller (LDC). Both the transmitted light through the optical fiber, and the scattered light from the microsphere at a right angle $\left(90^{\circ}\right)$ are detected. A polarizer is inserted between the microscope and photomultiplier tube (PMT) in order to separate the TE and the TM MDR's. The PMT signal is sent to a digital oscilloscope for signal monitoring and data acquisition. The optical power and the wavelength of the transmitted light are measured by an optical multimeter (OMM) with a silicon power/wavehead (PWH). Device control and data acquisition are performed with standard IEEE-488 GPIB interface. 


\section{EXPERIMENTAL RESULTS}

Fig. 2 shows the elastic scattering and the power transmission spectra obtained by temperature tuning of the DFB laser between $19{ }^{\circ} \mathrm{C}$ and $32{ }^{\circ} \mathrm{C}$ at a constant current of $31.2 \mathrm{~mA}$. This temperature ranges corresponds to a wavelength range between $811.9 \mathrm{~nm}$ and $812.7 \mathrm{~nm}$. The spectral resolution of the acquisition system is determined to be $0.04 \mathrm{~nm}$. Fig. 2(a) shows the elastic scattering spectrum of the TE MDR's, which is obtained through a polarizer with its polarization axis at $90^{\circ}$ to the incoming beam. Fig. 2(b) is obtained, when the polarizer is placed at $0^{\circ}$ to the incoming beam, corresponding to TM MDR's. MDR peaks and the associated dips are clearly observed in the elastic scattering and in the power transmission spectra, respectively. In the spectra of Fig. 2, the linewidth of the MDR's of higher intensity is approximately $0.03 \mathrm{~nm}(13 \mathrm{GHz})$, and the mode spacing of the higher intensity MDR's is measured to be $0.14 \mathrm{~nm}(63$ $\mathrm{GHz}$ ), which is consistent with the calculation based on the Mie scattering theory. The mode spacing is defined as the separation between the adjacent peak wavelengths of the same mode order (I) MDR's with subsequent mode numbers (n) [16]. It is important to note that the fractional depth of the dips in the transmission spectra is not the same for all MDR's. This is due to different Q-factors and coupling efficiencies of the MDR's. However, the relative depth of the transmission dips is maintained at each mode spacing. The broad Gaussian lineshape of the power transmission spectra results from the coupling geometry of the DFB laser to the optical fiber.
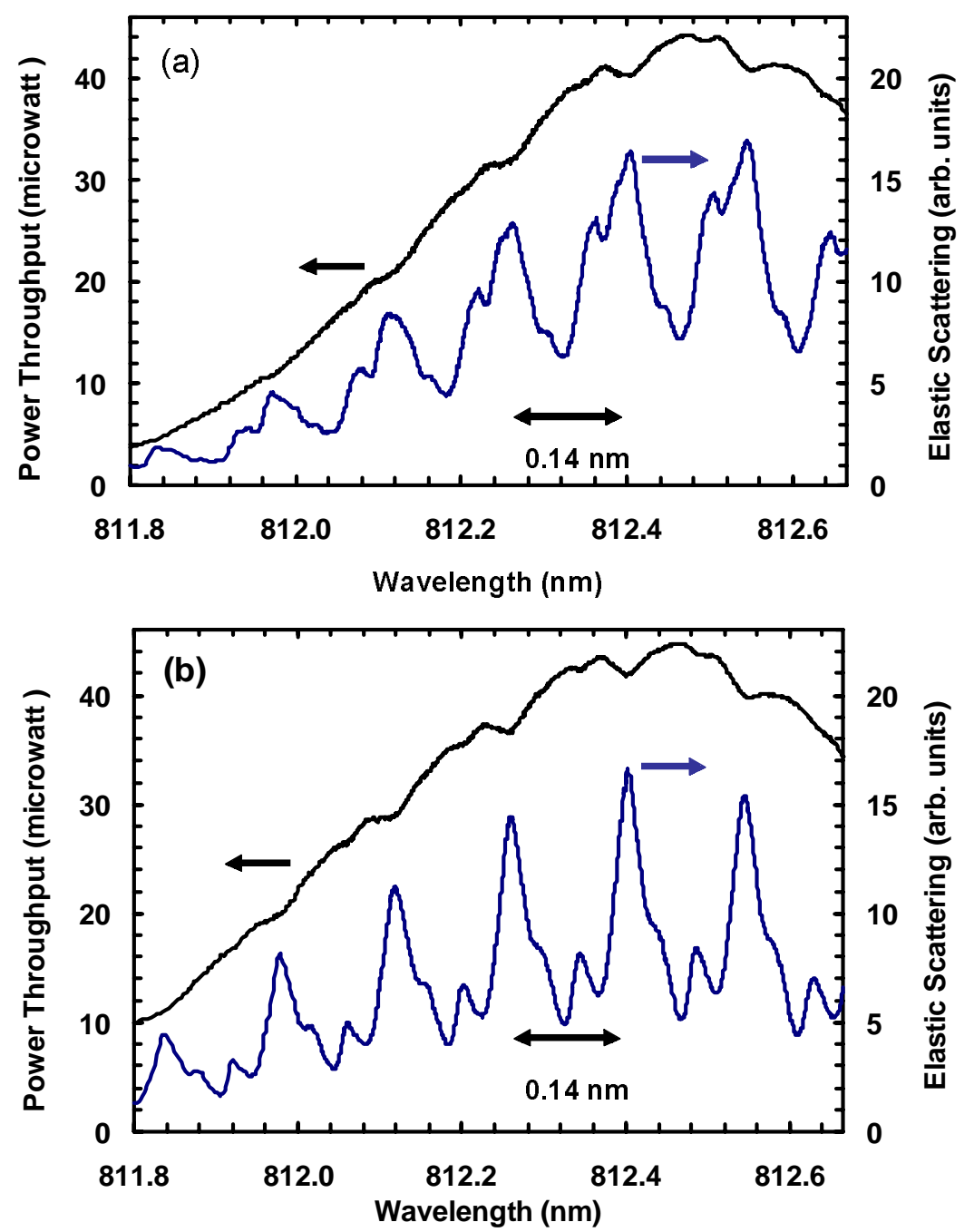

Figure 2. The transmission (upper curve) and the elastic scattering (lower curve) spectra when the polarizer is at (a) $90^{\circ}$ for TE and (b) $0^{\circ}$ for TM resonances, respectively. 
The measured Q- factor of the MDR's in the scattering spectrum is approximately on the order of $10^{4}$. The high Q factor MDR's are superimposed upon a background of low Q-factor MDR's. In practice, absorption, scattering on defects, scattering on impurities, residual surface roughness [17] are responsible for the measured losses corresponding to Qfactors due to the presence of small shape distortions, and surface irregularities of the particle [18]. The observed Qvalues should be compared with the values determined by $\mathrm{Q}^{-1}=\mathrm{Q}_{\mathrm{abs}}{ }^{-1}+\mathrm{Q}_{\mathrm{s}}^{-1}+\mathrm{Q}_{\mathrm{r}}^{-1}$, where $\mathrm{Q}_{\mathrm{r}}^{-1}$ corresponds to the intrinsic diffraction leakage, $\mathrm{Q}_{s}^{-1}$ the losses due to light scattering by surface roughness and Rayleigh scattering inside the sphere, and $\mathrm{Q}_{\mathrm{abs}}^{-1}$ the optical absorption loss.

\section{CONCLUSIONS}

The excitation of MDR's of glass microspheres has been demonstrated by using the OFHC. The MDR peaks in the elastic scattering spectra and associated dips in the transmission spectra are experimentally observed. The estimation of coupling efficiencies compares favorably with the experimentally measured values. With the proper necessary system design, it is possible to totally drop the selected MDR's power from the transmission spectrum.

\section{ACKNOWLEDGMENTS}

We would like to acknowledge the partial support of this research by the Scientific and Technical Research Council of Turkey (TUBITAK) Grant No: TBAG-1952 and the European Office of Aerospace Research and Development (EOARD) Grants No: F61775-01-WE062 and FA8655-02-M-4086.

\section{REFERENCES}

[1] L. Collot, V. Lefèvre-Seguin, M. Brune, J. M. Raimond, and S. Haroche, "Very high-Q whispering-gallery mode resonances observed on fused silica microspheres,” Europhys. Lett. 23, 327-334 (1993).

[2] A. Serpengüzel, S. Arnold, and G. Griffel, "Excitation of resonances of microspheres on an optical fiber,” Opt. Lett. 20 (7), 654-656 (1995).

[3] V. Lefèvre-Seguin and S. Haroche, “Towards cavity-QED experiments with silica microspheres," Materials Science and Engineering B 48(1-2), 53-58 (1997).

[4] V. Lefèvre-Seguin, "Whispering-gallery mode lasers with doped silica microspheres," Optical Materials 11 (2-3), 153-165 (1999).

[5] M. Kuwata-Gonokami and K. Takeda, "Polymer Whispering Gallery Mode Lasers," Optical Materials 9 (1-4), 12-17 (1998).

[6] S. M. Spillane, T. J. Kippenberg, and K. J. Vahala, "Ultralow-threshold Raman laser using a spherical dielectric microcavity," Nature 415 (6872), 621-623 (2002).

[7] V. S. Ilchenko, P. S. Volikov, V. L. Velichansky, F. Treussart, V. Lefèvre-Seguin, J. -M. Raimond, and S. Haroche, "Strain-tunable high-Q optical microsphere resonator,” Optics Communications 145 (1-6), 86-90 (1998).

[8] M. Cai, G. Hunziker, and K. J. Vahala, "Fiber-optic add-drop device based on a silica microsphere-whispering gallery mode system," IEEE Photon. Technol. Lett. 6 (6), 686-687 (1999).

[9] H. C. Tapalian, J. P. Laine, and P. A. Lane, “Thermooptical switches using coated microsphere resonators," IEEE Photon. Technol. Lett. 14(8), 1118-1120 (2002).

[10] S. Arnold, "Microspheres, photonic atoms and the physics of nothing," Am. Sci. 89, 414-421 (2001).

[11] B. R. Johnson, "Morphology-dependent resonances of a dielectric sphere on a conducting plane”, J. Opt. Soc. Am. A 11 (7), $2055-2064$ (1994).

[12] P. W. Barber, S. C. Hill, Eds., "Light Scattering by Particles: Computational Methods,” World Scientific, Singapore, 1990. 
[13] Richard K. Chang, Anthony J. Campillo, Eds., Optical Processes in Microcavities, World Scientific, Singapore, 1996; P. W. Barber and R. K. Chang, Eds., Optical Effects Associated with Small Particles, World Scientific, Singapore, 1988.

[14] A. Serpengüzel, S. Arnold, and G. Griffel, "Photonic atoms: Enhanced light coupling", in "Microcavities and photonic bandgaps: Physics and applications”, J. Rarity and C. Weisbuch, Kluwer Academic Publishers, Netherlands, 1995.

[15] G. Griffel, S. Arnold, D. Taskent, A. Serpengüzel, J. Connolly, and N. Morris, “Morphology-dependent resonances of a microsphereoptical fiber system,', Opt. Lett. 21 (10), 695-697 (1996).

[16] R. Jia, D. Jiang, P. Tan, B. Sun, J. Zhang, and Y. Lin, "Photoluminescence study of CdSexS1-x quantum dots in a glass spherical microcavity", Chin. Phys. Lett. 18 (10), 1350-1352 (2001).

[17] W. V. Klitzing, R. Long, V. S Ilchenko, J. Hare, and V. Lefèvre-Seguin, "Tunable whispering gallery modes for spectroscopy and CQED experiments", New J. Phys. 3 (14.1-14.14), (2001).

[18] J. P. Barton, "Effects of surface perturbations on the quality and the focused-beam excitation of microsphere resonance", J. Opt. Soc. Am. A 16 (8), 1974-1980 (1999). 